\title{
An Overview of the SOS High Temperature Pressure Sensor
} \author{
Xin $\mathrm{Li}^{1,2, \mathrm{a}}$, Defeng Liu ${ }^{1,2, \mathrm{~b}}$, Manguo Huang ${ }^{1,2, \mathrm{c}}$ and Wei Liu ${ }^{1,2, \mathrm{~d}}$ \\ ${ }^{1}$ Beijing Changcheng Aeronautic Measurement and Control Technology Research Institute, China \\ ${ }^{2}$ Aerospace Technology Key Laboratory of Special Sensing Technology for Condition Mornitoring, \\ China \\ alixin634@163.com, ${ }^{\mathrm{b}}$ liu_defeng@126.com, ${ }^{\mathrm{c}}$ mghuang2000@163.com, ${ }^{\mathrm{d}}$ Iw880623@126.com
}

Keywords: SOS. Silicon on Sapphire. High Temperature Pressure Sensor.

Abstract. SOS (Silicon on Sapphire) high temperature pressure sensor is a kind of strain pressure sensor with high performance. Since the sapphire has excellent properties in chemical stability, corrosion resistance, radiation resistance etc., the SOS high temperature pressure sensors are widely used in the industries of aerospace, ship, automobile, petroleum. In this paper, the technical advantages of SOS high temperature pressure sensor were summarized at first; then the manufacturing process and the research achievements at home and abroad of SOS high temperature pressure sensor were introduces; lastly, the development trend of SOS high temperature pressure sensor was demonstrated.

\section{Introduction}

High temperature pressure sensors aim at solving the problem about pressure measurement of various gas and liquid under the environment of high temperature (above $125^{\circ} \mathrm{C}$,). At present, the annual demand for high temperature pressure sensors is about 4 million. With the development of national economy and national defense modernization, the market demand of high temperature pressure sensors will be increased by $10 \%$ to $32 \%$ per year [1].

In the field of aviation, the demand of high temperature pressure sensor is particularly urgent. Especially in many key parts of aircraft engine, such as hydraulic system, cooling system, engine gas path, compressor and so on, are usually working under harsh environment with high temperature, high pressure and strong vibration. Therefore, it is necessary to use high temperature pressure sensors to monitor the pressure changes in real time, in order to perceive the unexpected change of pressure as soon as possible, and avoid the occurrence of malignant accidents.

The conventional pressure sensors cannot realize the pressure measurement of the aircraft engines, high temperature wells, boilers, which work under high temperature conditions. Whereas the SOS high temperature pressure sensors have the good properties such as radiation resistance, corrosion resistance, high impedance and low power consumption. And they can revoid the technical problems of the conventional ones such as low performance stability, poor environmental testing adaptability, short service life and low reliability. As a result, the SOS high temperature pressure sensors have received more and more attention of experts and scholars at home and abroad [2,3].

\section{Technical Advantages of the SOS}

SOS(silicon on sapphire) high temperature pressure sensor is made based on the piezoresistive effect of silicon. The single crystal silicon forms a strong molecular bond to a sapphire substrate, utilizing MEMS processing technology connect the four strained silicon resistors connected in a Wheatstone bridge configuration. According to the Wheatstone principle:

$$
\mathrm{V}_{0}=\frac{R_{1} R_{4}-R_{2} R_{3}}{R_{1}+R_{2}+R_{3}+R_{4}} I_{0}
$$

Where $R_{1}, R_{2}, R_{3}$ and $R_{4}$ represent the four strain gauges of Wheatstone principle, and $I_{0}$ represents the current of the constant-current source. When subjected to external forces, $R_{1}$ and $R_{4}$ will 
have a positive increment $\Delta R_{1}$ and $\Delta R_{4}$ due to the piezoresistive effect, while $R_{2}$ and $R_{3}$ will have a negative increment $\Delta R_{2}$ and $\Delta R_{3}$. When $R_{1}=R_{2}=R_{3}=R_{4}=R$, the voltage can be expressed as:

$$
\mathrm{V}_{0}=\Delta \mathrm{RI}_{0}
$$

Therefore, through the Wheatstone bridge, the non-electricity pressure can be transformed into electricity by the use of piezoresistive effect of silicon resistors.

The conventional pressure sensors are generally based on the diffuse silicon semiconductor sheet as a force-sensitive strain gauge, which the P-type silicon layer is epitaxially grown on the N-type silicon substrate. As the silicon substrate and the sensing resistor are isolated relying on the P-N junction, leading to a large leakage of current and a low operating temperature, the performance of sensors decreases sharply above $120^{\circ} \mathrm{C}$, which greatly limits the application of the conventional ones. The SOS sensors do not use the PN junction isolation, instead of using the dielectric isolation. Due to the good insulation of sapphire, the single-crystal silicon strain gauges grown on it are completely independent of each other in terms of electrical performance. The temperature-change insensitivity of sapphire and the high sensitivity of silicon are combined neatly, and the drift problem caused by the different junction leakage is eliminated. So the SOS pressure sensors can maintain long-term stability under high temperature condition.

What's more, for the traditional pressure sensors, the strain resistance and the strain gauge are connected by the means of bonding or common deposition. Since the silicon is grown onto the surface of the sapphire, it is incredibly stable and exhibits virtually no hysteresis. With its outstanding insulation properties, the sapphire protects the strain gauge from electromagnetic pulse radiation, thereby allowing the sensor to operate over a very wide temperature range without loss of performance. It can endure high over pressures and provides outstanding corrosion resistance. The sapphire's excellent elasticity ensures high repeatability- a highly desirable characteristic for sensors.

In addition, stainless steel, Hastelloy and other metal material or silicon semiconductor material used in conventional pressure sensors is susceptible to corrosion in acid and alkali medium during long-term operation. And some even can't be used in strong acid or alkali environment. And the metal materials are prone to creep under high pressure, accompanied by serious hysteresis and nonlinear phenomena. However, sapphire material has a strong acid and alkali corrosion resistance, almost no solution can cause corrosion damage to it. Therefore, the sensor can be directly contacted with the measured medium without the protection of the isolating diaphragms or fillings, which avoids the measurement error due to the slight deformation of the diaphragms or fillings. Meanwhile sapphire material almost has no hysteresis. Because of the specialty of sapphire crystal lattice, the deformation of sapphire subjected to pressure is proportional to the external force until the moment of rupture. After removing or reducing the force, the sapphire can be able to restore the original shape fully complied with the Hooke's law. Table 1 shows the inherent characteristics of the SOS pressure sensor and its corresponding advantages.

Table 1 Advantages of the SOS

\begin{tabular}{|c|c|}
\hline The Inherent Characteristics & The Advantages \\
\hline Sapphire is composed of single crystal insulator element & No hysteresis, fatigue or creep \\
\hline Sapphire is harder than silicon & Good reproducibility \\
\hline SOS sensing elements do not use P-N junction & $\begin{array}{c}\text { No dark current. High accuracy under } \\
\text { high temperature environment }\end{array}$ \\
\hline $\begin{array}{c}\text { The sensing element and elastic element are connected } \\
\text { through high temperature sintering }\end{array}$ & Good vibration resistance \\
\hline Sapphire has chemical stability & Good corrosion resistance \\
\hline
\end{tabular}

For the above reasons, using SOS structures in piezoresistive transducers has proven to be so successful that the technology can be widely applicable to complex and special environment in an 
overwhelming majority of pressure measurement. And the maximum operating temperature of the SOS sensor can reach $550^{\circ} \mathrm{C}$.

\section{Manufacturing process of the SOS}

Due to the high hardness and corrosion resistance of sapphire material, sapphire film is produced mainly by mechanical methods. The key technologies include the hetero epitaxial growth, ohmic contact, thinning and separation of pressure diaphragm, reliable transfer between internal and external wires, reduction the switching stress between the sapphire and metal or ceramic, inhibition of self-doping, etc.

In general, the sensing component is produced by the means of microelectronics mechanical processing technology, and the general process is:

Selection of films - Diffusion - Silicon resistance lithography - Aluminum steamed - Electrode lithography - Alloy - Testing

And the sensor is assembled by the means of high temperature sintering or argon arc welding. The general process is:

Isolation and filter-Aluminum dislodged - Sintering-Electrode steamed - Argon arc welding Package-Testing - Temperature compensation - Calibration

It should be noted that during the lithography, the accuracy of the silicon resistance position is directly affected by the aiming precision, and the quality of the resistance is directly affected by the focusing accuracy. When the depth of field in focus is too long (more than $2 \mu \mathrm{m}$ ), the sealing surface between sapphire and metal is easy to damage. When the depth of field in focus is too short (less than $2 \mu \mathrm{m}$ ), etching of silicon resistance is not enough. It's mentioned in [6] that the usage of multiple frequency technique can improve the accuracy of aiming and focusing, changing the infrared laser of $1.06 \mu \mathrm{m}$ wavelength into green laser of $5300 \AA$ wavelength..

\section{Research Status of the SOS}

The study of the SOS high temperature pressure sensor is initially for the purpose of military applications. The search of the SOS begins from 60s in the former Soviet Union (now Russia). Early in 70s-80s, Russia Microelectronic Sensor Industrial Group (short in MIDA) has been carrying on in-depth research about the hetero epitaxial semiconductor structure - "Silicon on sapphire", and obtained a lot of scientific research results. The SOS sensors of MIDA are at the advanced level in the world, and occupy a large market share. They are widely acclaimed because of the high measuring accuracy, good repeatability, large overload pressure, etc. Especially, the MIDA - GP - 55 Titanium/Silicon on Sapphire sensor (shown in Fig. 1) can work at $550^{\circ} \mathrm{C}$, measure to $60 \mathrm{MPa}$ with a comprehensive error of $0.5 \%$ F.S, and satisfy the high temperature pressure measurement during the engine test.

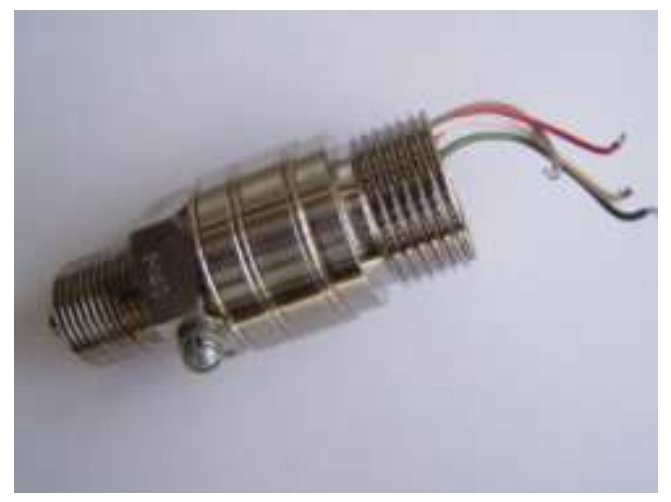

Fig. 1 The SOS sensor of MIDA

Yu. A. vas' KOV et al. from Russia have studied on how to utilize the analog-to-digital converters (ADCs) and microcontrollers (MCUs) to reduce the influence of noises [8] and temperature [9] on 
measurement accuracy of the SOS sensors. The experimental results testify that by the use of a 16-digit $\Delta \Sigma$--ADC AD7798, the reduced measurement error of pressure values is almost independent from the filtering methods for the signals supplied by the primary transducer and constitutes less than $0.075 \%$. The error introduced by the sample span of ADC codes is random and does not exceed $0.005 \%$. The obtained result of reduced measurement error is shown in Fig.2.

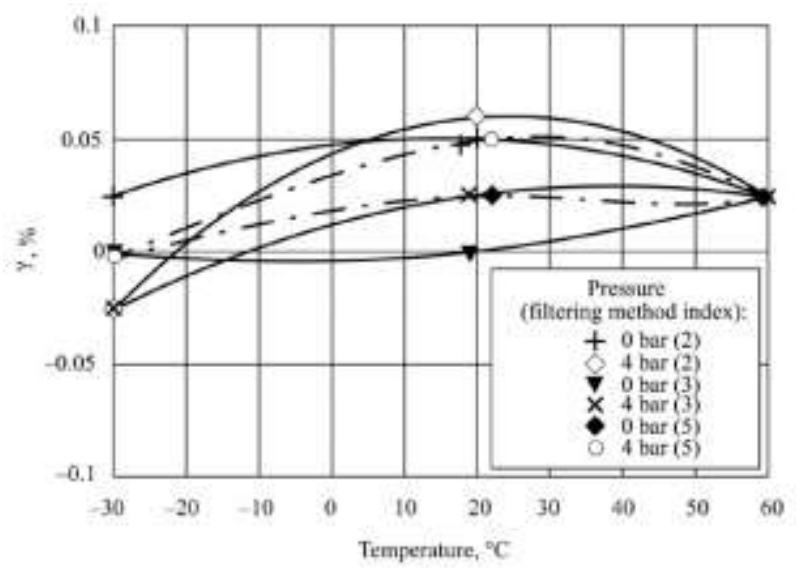

Fig.2 The reduced measurement error of pressure under different filtering methods

The United States and other developed countries have been studied on the SOS sensors for many years. With the relatively mature MEMS technology, the performance of the chip is much more stable, and the work is also more detailed. As a result, the SOS sensors produced have good performance and reliability. In the United States occupied dominant position are Sensonetics company's products.

Their SOS sensors have a pressure measurement range from 0 to 0.5 to $0 \sim 50000$ PSI, and a working temperature range from $-20^{\circ} \mathrm{C}$ to $350^{\circ} \mathrm{C}$. The productions have been applied in $\mathrm{F} 117$ stealth bombers, MIZA tanks, tanks, deep water bombs, etc. [10].

The No.49 Institute of Electronic Information Industry in China has introduced the new dual-mode structure of Titanium / Silicon on Sapphire high temperature pressure sensors in the eleventh five year period. After along time of technical accumulation, it already has a complete production line today. And the SOS sensors produced can measure pressure from $0 \sim 0.1 \mathrm{MPa}$ to $0 \sim 250 \mathrm{Mpa}$. The operating temperature range is $-20^{\circ} \mathrm{C} \sim 350^{\circ} \mathrm{C}$. The accuracy is better than $0.1 \%$ [11]. The structure of the SOS sensor produced by No.49 Institute is shown in Figure 3. The No.634 Institute of AVIC has developed the SOS high temperature pressure sensor specialized in the health management of aviation aircraft hydraulic system. The measured pressure range is $0 \sim 28 \mathrm{MPa}$. The operating temperature range is $-20^{\circ} \mathrm{C} \sim 250^{\circ} \mathrm{C}$. And the comprehensive error is better than $0.5 \% \mathrm{FS}$.

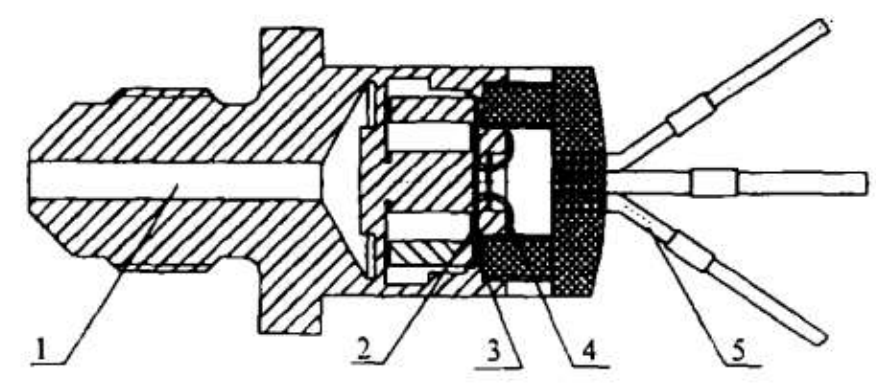

Fig.3 The structure of the SOS sensor

\section{Summary and Prospect}

The SOS high temperature pressure sensor has many excellent characteristics, such as chemical stability, corrosion resistance, radiation resistance, and so on. It has broad application prospects in civil industry and national defense industry. However, because of the high cost of the preparation of sapphire single crystal, the difficult and complex processing, the low yield of strain gauges, the production and engineering application of the SOS sensors is limited. What's more, due to 
temperature cross-sensitivity phenomenon under high temperature environment, the compensation of sensitivity and linearity drift caused by temperature change has become more and more concerned. With the continuous development of novel semiconductor materials and MEMS processing technology, the problems such as structural design, membrane preparation, hot melt processing, etc. will be gradually resolved. In the future, it is expected to realize large-scale engineering application of the SOS high temperature pressure sensor.

\section{Acknowledgements}

This work was financially supported by the Aviation Science Foundation (2014ZD34002) and Technology Innovation Foundation of AVIC (2013F63447R).

\section{References}

[1] Tianzhao Pang, Zinlin Yan, Fei Tang, Xiaohao Wang. Recent Development and Future Perspective of Silicon Carbide High-Temperature Pressure Sensor. Journal of Noise and Vibration Control, 2010(1):170-174. (In Chinese)

[2] Stuchebnikov V M. SOS Strain Gauge Sensors for Force and Pressure Transducers. Journal of Sensors and Actuators A, 1991, 28 (3):207-213.

[3] V.M.Stuchebnikov. Silicon-on-Sapphire Structures as a Material for Piezoresistive Mechanical Transducers. Journal of Communications Technology and Electronics, 2005, 50(6):622-637.

[4] Xiguang Yuan, in: Sensor Technical Manuals, National Defence Industry Publication, Beijing, in press. (In Chinese)

[5] Shirong Zhang, Bin Wen, Guanghao Cui. A Kind of SOS High Temperature Pressure Transducer with Double Membrance, Journal of Natural Science of Heilongjiang University, 2001(4):56-62. (In Chinese)

[6] Lei Duan, Technology of New Wide-Temperature-Range Pressure Sensor, Journal of Instrument Technique and Sensor, 2009(12):17-18. (In Chinese)

[7] G.G.Iordan. In Microelectronic Transducers for Measuring Mechanical and Thermal Properties (MDNTP, Moscow, 1980) p.3. (In Russian)

[8] Yu. A. Vas'kov, G. A. Emel'yanov. Intelligent Pressure Sensors Based on Silicon-on-Sapphire Structures: The Influence of Noises on Measurement Accuracy. Journal of Automation and Remote Control, 2014(11):2086-2090.

[9] Vas'kov, Yu.A., Emel'yanov, G.A., Kozlov, A.I., A Digital Correction Method for the Temperature Error of Pressure Sensors Based on Silicon-on-Sapphire Structures, Radioelektronnaya tekhnika (Electronic Radio Equipment), Ulianovsk: Ulianovsk. Gos. Tekh. Univ., 2009: 72-79.

[10] Chengchen Gao. The Research Status of Silicon-on-Sapphire High Temperature Sensor, Proceedings of the Symposium on National Mechanical Quantity Sensors: Test and Metrology, 2000: 24-27. (In Chinese)

[11] Hua Wen, Aiping Zhang, Feng Zhang. Design of High Temperature Wide Range Silicon - on Sapphire Pressure Sensor, Journal of Transducer and Microsystem Technologies, 2007, 26(12): 97-99. (In Chinese) 